\title{
THE EFFECT OF TIP RADIUS ON NANO-INDENTATION HARDNESS TESTS
}

\author{
CHUNG-JEN LU† and D. B. BOGY \\ Department of Mechanical Engineering, Computer Mechanics Laboratory, University of \\ California at Berkeley, CA 94720 , U. S. A
}

(Received 13 May' 1994; in revised form 2 August 1994)

\begin{abstract}
Sub-micron indentation hardness tests on silicon and aluminum, both in their bulk forms and in layer-substrate combinations, are simulated by the finite element method using a blunt indenter. The blunt indenter is modeled by a cone with a spherical cap with various radii. Emphasis is placed on the effect of the tip radius on the hardness tests. Two methods for the determination of the contact area are employed: one measures the contact area from the residual indentation; the other uses the plastic depth and the area-depth relationship of the indenter. Indentation tests on bulk materials show weak tip and indentation size effects. For an aluminum layer on a silicon substrate, the hardness is relatively insensitive to the tip radius. On the contrary, for a silicon layer on an aluminum substrate, the hardness is much more sensitive to the tip radius. In order to obtain the hardness of this layer-substrate combination without the influence from the substrate, the tip radius to layer thickness ratio should be less than 1.25 .
\end{abstract}

\section{INTRODUCTION}

Thin films have been extensively used to enhance the tribological performance of contact surfaces. With the progress of deposition techniques, the thickness of thin films has decreased to a few tens of nanometers. Knowledge of in situ mechanical properties of thin films is helpful for selecting proper materials and deposition conditions. The indentation hardness test may be the most convenient method to determine the mechanical strength of thin films.

Several researchers have reported work on nano-indentation hardness tests (Gane and Bowden, 1968 ; Pethica et al., 1983 ; Wierenga and Franken, 1984 ; Pharr and Oliver, 1989; Lu et al., 1994). Nano-indentation tests on different materials show a common indentation size effect : the hardness increases with decreasing indentation depth. For a perfectly sharp indenter on a homogeneous material, the hardness is independent of the indentation depth due to the geometric similarity. However, in practical nano-indentation tests, the fact that the indenter has a nonzero tip radius cannot be neglected (Doerner and Nix, 1986, Lu et al., 1994). The observed size effect may result from the fact that the contact is not fully plastic due to the nonzero radius at small indentation depths. Another interesting problem that needs to be studied is the effect of tip radius on the measured hardness of layered media.

Finite element analysis is capable of giving insight into the indentation process. Several researchers have presented simulations of indentation tests with either a spherical or a cylindrical indenter (Akyuz and Merwin, 1968; Hardy et al., 1971; Follansbee and Sinclair, 1984; Komvopoulos, 1989; Kral et al., 1993). Emphasis is put on the stress and strain distribution in the specimen. Bhattacharya and Nix (1988a,b, 1991) simulated indentation tests on bulk materials and layered media with different combinations of layer and substrate materials. The effect of material properties on the predicted hardness was investigated. They used a rigid, perfectly sharp conical indenter. Shih et al. (1991) compared the experimental load-displacement curve with simulation results using conical indenters with different tip radii to determine the tip radius of the indenter used in experiments.

In the present study, we extend the work of Bhattacharya and Nix to indenters with spherical tips of different radii. Two methods are used to determine the hardness. The effect

† Presently: Associate Professor, National Taiwan University, Taipei, Taiwan. 


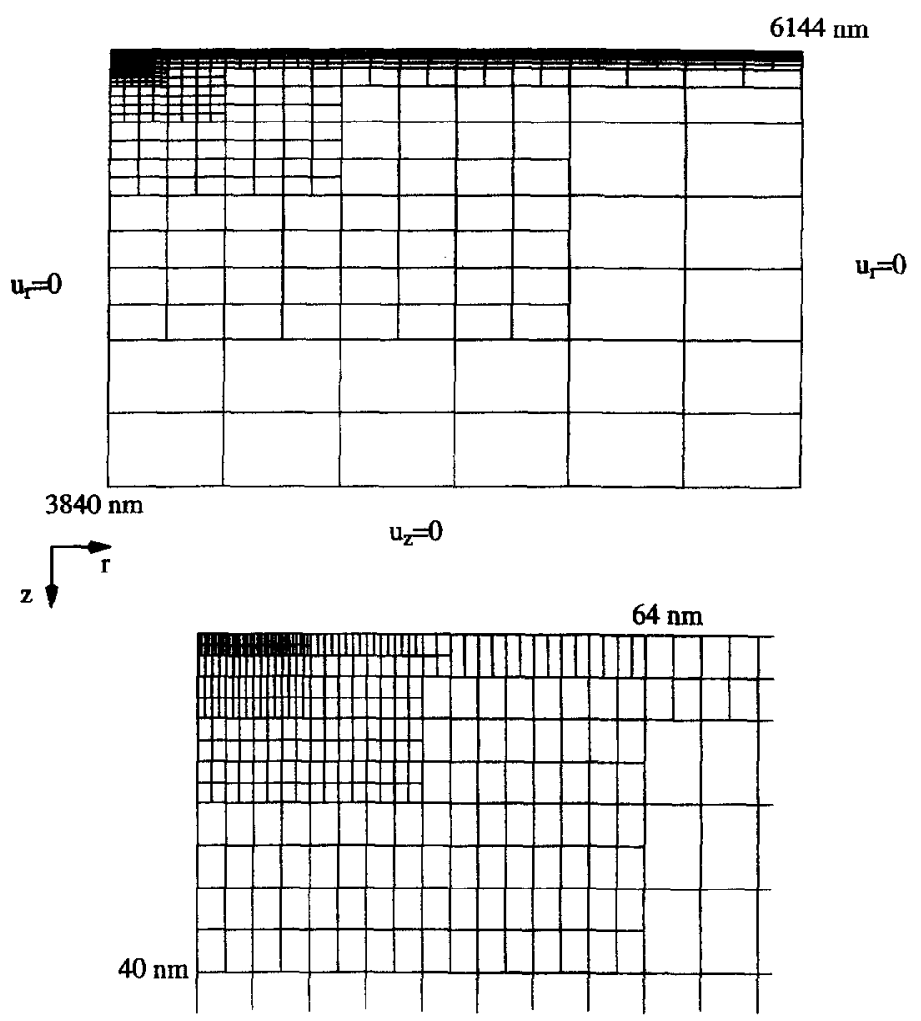

Fig. 1. Finite element model.

of tip radius and indentation depth on the hardness tests is investigated. The limitation on tip radius for obtaining the hardness of the layer is presented.

\section{MODELING PROCEDURES}

\subsection{Finite element model}

In this study, rigid conical indenters with different tip radii are used to simulate indentation hardness tests. The indenter and the specimen are treated as bodies of revolution to avoid the difficulty of modeling the real pyramidal indenter with nonzero tip radius. The large strain elastic-plastic capability of the ABAQUS (1989) code was employed for the finite element simulation. The simulation process is basically the same as used by Bhattacharya and Nix $(1988 \mathrm{a}, \mathrm{b})$ except that they used perfectly sharp indenters and a different mesh.

Figure 1 shows the finite element mesh, which consists of 1136 4-node quadrilateral axisymmetric elements and 1328 nodes. The analysis is intended for nano-scale indentation and is not made dimensionless. The $z$ - and $r$-ranges of the mesh are $3840 \mathrm{~nm}$ and $6144 \mathrm{~nm}$, respectively. A magnified view of the fine meshes in the range of $0<r<64 \mathrm{~nm}$ and $0<z<40 \mathrm{~nm}$ is shown in the bottom of Fig. 1. The smallest element size along the $r$-axis is $0.5 \mathrm{~nm}$ to obtain an accurate estimate of the radius of the contact area and to describe the associated deformation and stress gradients. The meshes become coarser with distance away from the indenter. When two elements are connected to a single element, the middle node is constrained to lie on the straight line determined by the two corresponding end nodes. Symmetry properties are used to simplify the boundary conditions. The vertical boundaries of the mesh are constrained against displacement in the $r$-direction, and the bottom against displacement in the $z$-direction. Further enlargement of the mesh dimensions and changes of the boundary conditions at the right side produce no significant effect on the result. 


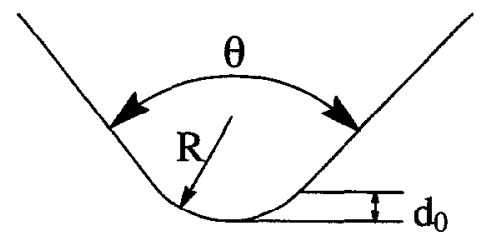

Fig. 2. Schematic of an indenter.

Figure 2 shows a schematic diagram of a typical indenter, which consists of a sphere with radius $R$ and a cone with included angle $\theta$. Three different radii, 0,100 and $200 \mathrm{~nm}$, are used to study the effect of the tip radius on the indentation tests. The included angle is chosen to be $138^{\circ}$ in order to compare with the results of Bhattacharya and Nix (1988a). For a perfectly sharp cone, this included angle also generates the same displaced volume to depth ratio as that of a Vickers indenter. As can be seen from the figure, when the indentation depth is less than $d_{0}$, the indenter is simply a sphere. On the other hand, for indentation depths much larger than $d_{0}$, the indenter can be treated as a perfectly sharp cone. The rigid indenter is modeled by a total of 1053 -node interface contact elements. Two nodes of each contact element correspond to surface nodes of the media in the range of $0<r<448$. The third node is a common node assigned to the rigid indenter, which can move only in the $z$-direction.

To simulate an indentation process, a displacement in the $z$-direction is imposed on the master node of the indenter; this causes the indenter to penetrate into the specimen. The change of the separation between the indenter and the specimen surface nodes, such as any gap closure during loading and gap opening during unloading, is monitored by the program automatically. For a given indentation, the corresponding load is determined by summing the reaction forces at the contact nodes. The interface between the indenter and the specimen is assumed to be frictionless because of the marginal effect of friction in normal contact (Komvopoulos, 1989).

Two different materials, aluminum and silicon, are used in this analysis. The constitutive model assumed here is the elastic-perfectly plastic von Mises material. The material properties used in the calculations are given in Table 1 . These materials were also considered by Bhattacharya and Nix $(1988 \mathrm{a}, \mathrm{b})$ with a perfectly sharp conical indenter. Besides homogeneous specimens, these materials are also combined to form a hard layer on a soft substrate ( $\mathrm{Si}$ on $\mathrm{Al}$ ) and vice versa. Three different layer thickness values, 20, 40 and 80 $\mathrm{nm}$, are considered here.

\subsection{Verification of the FEM model}

To examine the suitability of the finite element model, elastic indentations between a rigid sphere and an aluminum half-space were performed and the surface pressure was compared with the Hertz solution. In the finite element simulation, a $5 \mathrm{~nm}$ indentation was imposed on the spherical indenter with a radius of $1000 \mathrm{~nm}$. The corresponding Hertz solutions of the contact radius and maximum contact pressure are $70.7 \mathrm{~nm}$ and $3.834 \mathrm{GPa}$, respectively. As can be seen from Fig. 3, the finite element result matches with the Hertz solution fairly well.

In addition, elastic-plastic indentation with a rigid cone on an aluminum half-space was performed to further verify the finite element model. The comparison of the loaddisplacement curve for a perfectly sharp cone with the result obtained by Bhattacharya and Nix (1988a), indicated by circular dots, is shown in Fig. 4. Good agreement between these

Table 1. Elastic and plastic properties of aluminum and silicon

\begin{tabular}{lccc}
\hline Material & $\begin{array}{c}\text { Young's modulus } \\
(\mathrm{GPa})\end{array}$ & $\begin{array}{c}\text { Yield strength } \\
(\mathrm{GPa})\end{array}$ & Poisson's ratio \\
\hline Aluminum & 75.9 & 0.485 & 0.33 \\
Silicon & 127 & 4.4 & 0.278 \\
\hline
\end{tabular}




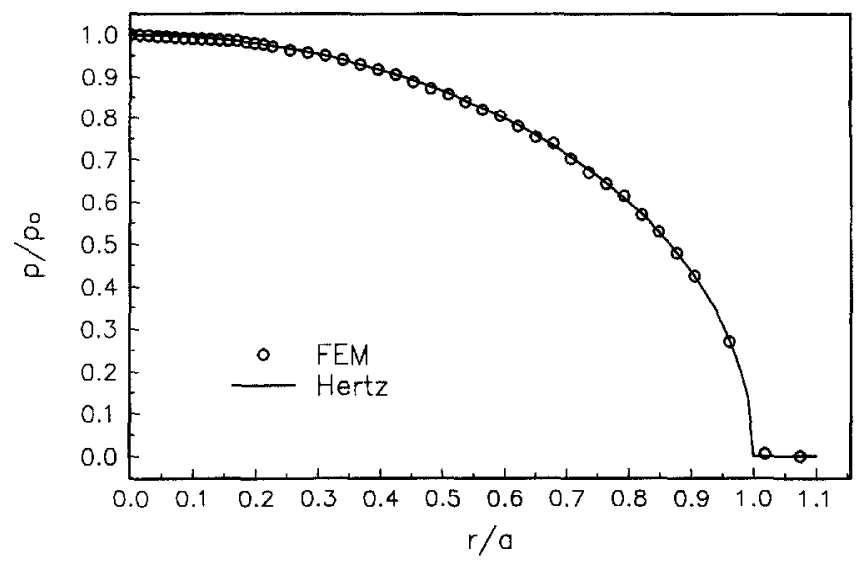

Fig. 3. Comparison of finite element result with Hertz solution.

two results can be clearly seen from the figure. Therefore, the finite element model is accepted as appropriate for the simulation of indentation hardness tests.

\section{HARDNESS MEASUREMENT}

Two methods have been developed to measure the sub-micro hardness. The first one uses a depth-sensing instrument to measure the indentation force and depth simultaneously (Pethica et al., 1983; Yanagisawa and Motomura, 1987). In order to obtain the hardness without imaging the indentation, TEM and replica technology are used to obtain the crosssectional area versus depth relation of the indenter (Pethica et al., 1983; Doerner and Nix, 1986). With this information, the hardness can be obtained from the load-displacement curve if a proper depth is chosen to determine the contact area. Figure 5 shows a typical load-displacement curve obtained from the finite element simulation of indentation on silicon with a perfectly sharp cone. Among the three depths, indicated on the figure as maximum depth, plastic depth and residual depth, Doerner and Nix (1986) found that the contact arca based on the plastic depth is closer to the real contact area at the maximum load than those based on the maximum and residual depths. The plastic depth is determined from the load-displacement curve by first fitting a line tangent to the unloading curve at the maximum load. The interception of the line and the $x$-axis is the plastic depth. A detailed description of the theory behind this procedure was given by Oliver and Pharr (1992). In this simulation, the cross-sectional area of the indenter, based on the plastic depth, is referred to as the plastic depth area for convenience.

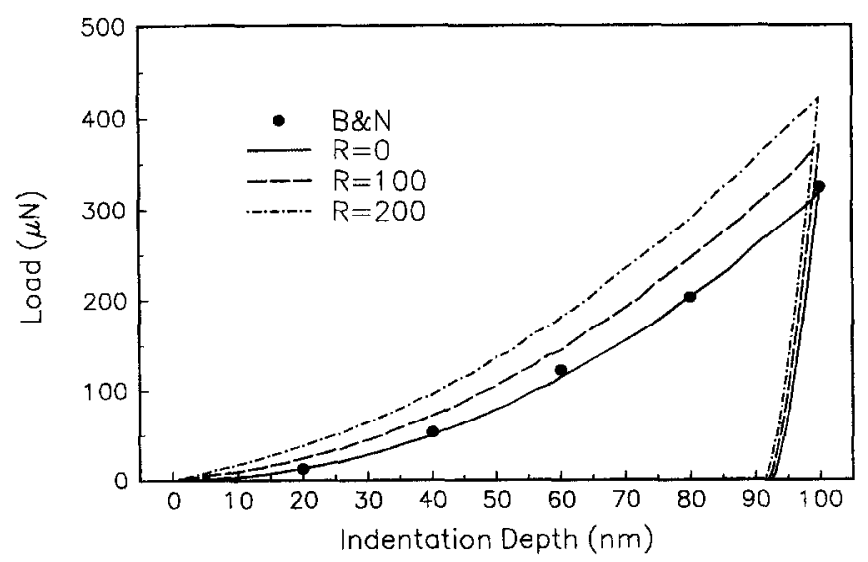

Fig. 4. Load-displacement curves on aluminum with different tip radii, dots indicate results from Buhattacharya and Nix (1988a). 


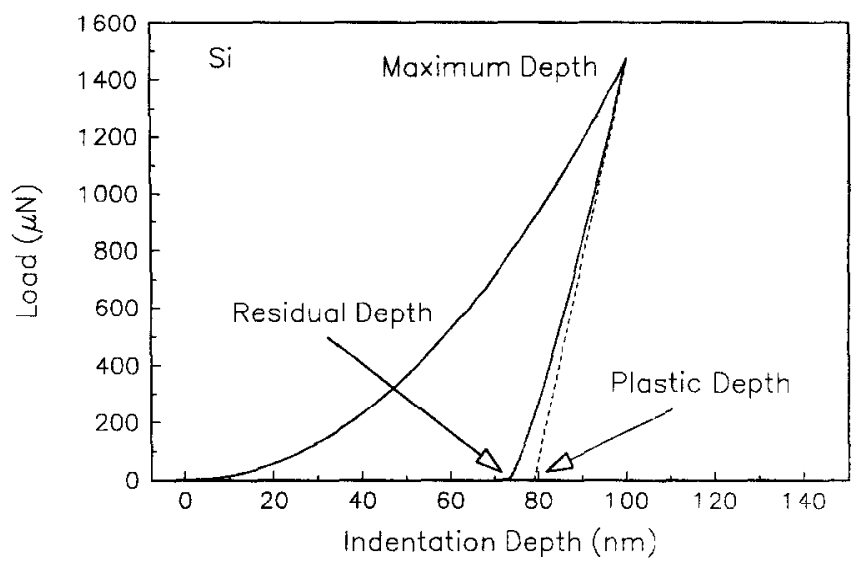

Fig. 5. Load displacement curve on silicon with tip radius of zero to illustrate the definition of plastic depth.

The other method, employing the modern Scanning Probe Microscopy (SPM) technology, uses the same tip to first indent the surface under a well controlled load and then scan the surface to image the indentation (Lu et al., 1994). With the high resolution of the SPM, better than one nanometer, the residual indentation area can be very accurately measured. The hardness is defined as the ratio of the load to the projection area of the residual indentation. To simulate this process, the interception of the $r$-axis (the line $z=0$ ) and the residual displacement of the indentation is used to calculate the area, referred to as residual area hereafter.

\section{RESULTS AND DISCUSSION}

\subsection{Homogeneous half-space}

Load-displacement curves for indentations on aluminum using indenters with various tip radii are shown in Fig. 4. For a given indentation depth, the applied load increases with the tip radius, as expected. The residual and plastic depths are both close to the maximum depth in these cases, which indicates relatively small elastic recovery during the unloading process. This is in contrast to the indentation on silicon (Fig. 5), where a larger elastic recovery is shown. The difference in elastic recovery between aluminum and silicon is evidently due to their different Young's moduli to yield stress ratios, 29 and 156 for silicon and aluminum, respectively.

Residual indentation profiles using different indenters with maximum depths of 10 and $100 \mathrm{~nm}$ for $\mathrm{Al}$ and $\mathrm{Si}$ are shown in Figs 6 and 7, respectively. With maximum depth of 10 $\mathrm{nm}$, Figs 6(a) and 7(a), the residual area increases dramatically with tip radius, while the residual depth decreases with increasing tip radius. On the other hand, with a maximum depth of $100 \mathrm{~nm}$, the residual displacements of different tip radii are close to each other. Therefore, both the residual area and depth are sensitive to the tip radii at small indentation depths.

Hardness versus indentation depth curves obtained from the residual area for aluminum with various tip radii are shown in Fig. 8(a). For a perfectly sharp cone, the hardness is essentially independent of the indentation depth. For indenters with a nonzero tip radius, the hardness is less at smaller indentation depths. In addition, as can be seen from the figure, for indentation depths less than $40 \mathrm{~nm}$, the hardness decreases somewhat with increasing tip radius. However, the variation is less than $10 \%$. The hardness for indentation depths larger than $60 \mathrm{~nm}$ is not sensitive to either the tip radius or the indentation depth. The hardness obtained from the plastic depth area presents the same tendency [Fig. 8(b)]. For the same tip radius and indentation depth, the hardness values obtained from these two methods are close to each other. Similar features can also be found from the results of indentation hardness tests on silicon using tips with different radii (Fig. 9). Comparing Figs 


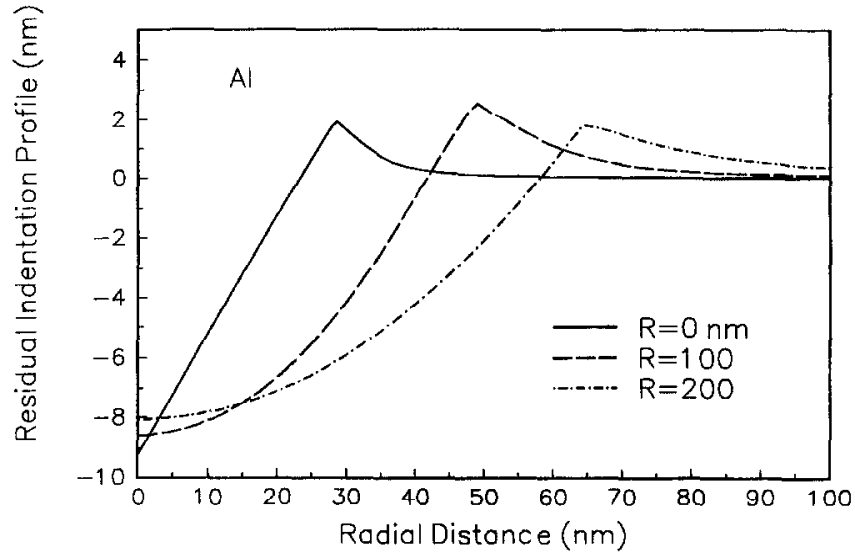

(a)

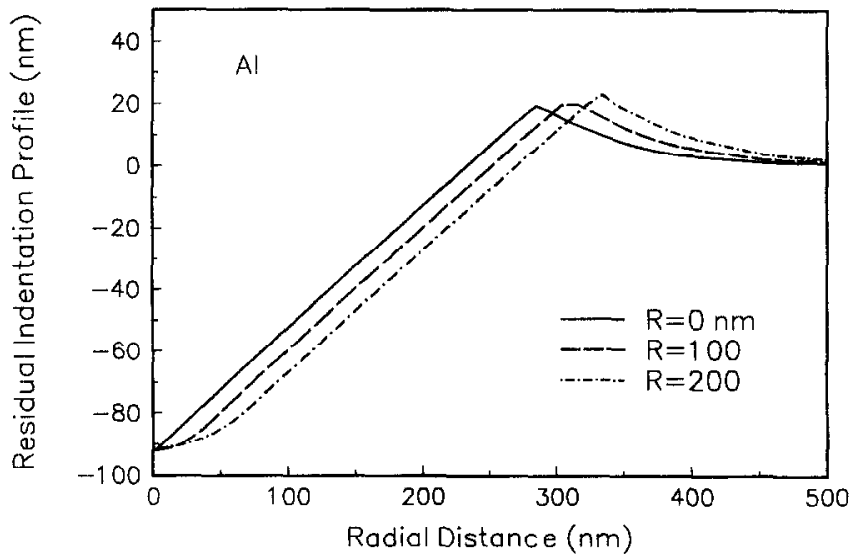

(b)

Fig. 6. Residual indentation profile of Al with various tip radii at maximum indentation depths of (a) $10 \mathrm{~nm}$ and (b) $100 \mathrm{~nm}$.

9(a) and 9(b), we see that the hardness values obtained from the residual area are somewhat less than those obtained from the plastic depth area.

Indentation tests by Tabor (1951) on work-hardened copper using a spherical indenter of $10 \mathrm{~mm}$ diameter show that the hardness increases with the chordal diameter of the indentation. It was also found that, for spherical indenters with different radii, the hardness increases with the ratio of the contact radius to the tip radius. The present finite element results show the same tendency at small indentation depths, where the indenter is simply a sphere. For larger indentation depths, the effect of the tip radius is negligible and the indenter can be treated as a cone. Therefore the hardness is independent of the indentation depth due to the geometrical similarity. On the other hand, nano-indentation hardness tests performed by several researchers (Gane and Bowden, 1968; Pethica et al., 1983; Pharr and Oliver, 1989) on various materials show that hardness increases with decreasing indentation depth. This opposite tendency to the present finite element result may be due to different deformation mechanisms in the experimental nano-indentation test and the simulation. As suggested by Pharr and Oliver (1989), nano-scale plasticity may take place by nondislocation mechanisms. Molecular dynamics simulations by Belak et al. (1993) show that nano-scale plastic deformation due to point indentation takes place through the creation and motion of point defects.

\subsection{Layered media}

Two different cases are considered, one is a soft layer on a hard substrate (aluminum on silicon), the other is a hard layer on a soft substrate (silicon on aluminum). Three layer 


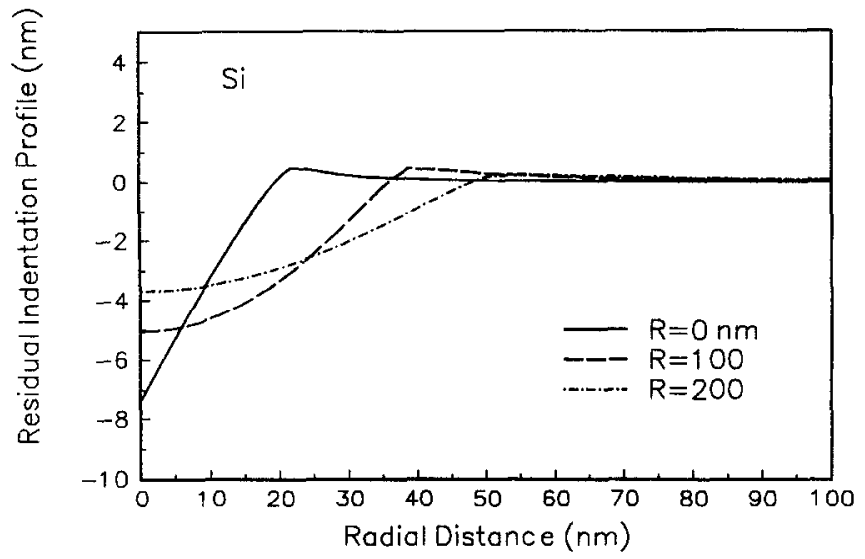

(a)

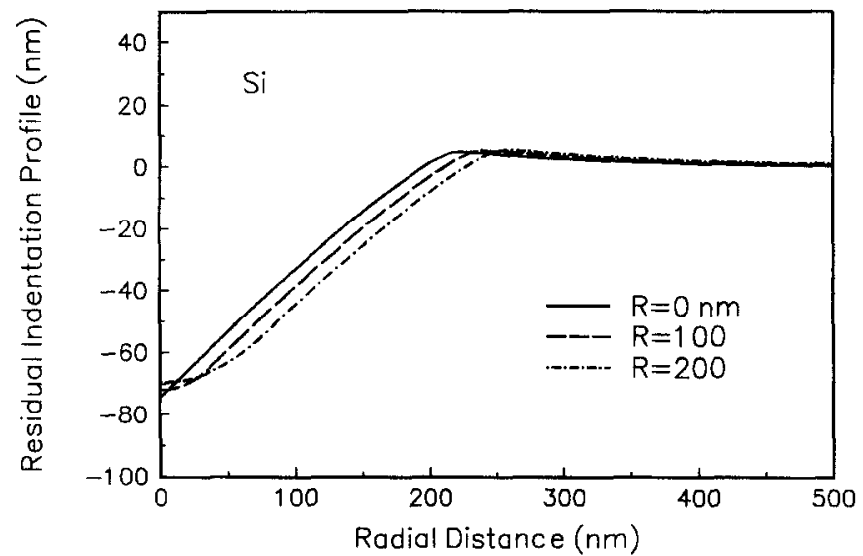

(b)

Fig. 7. Residual indentation profile of $\mathrm{Si}$ with various tip radii at maximum indentation depths of (a) $10 \mathrm{~nm}$ and (b) $100 \mathrm{~nm}$.

thickness values, 20,40 and $80 \mathrm{~nm}$, are used to give a variety of tip radius to layer thickness ratios of $0,1.25,2.5$ and 5.0. Figure 10 shows residual indentation profiles on a $20 \mathrm{~nm}$ thick aluminum layer with maximum depths of 2.5 and $10 \mathrm{~nm}$, respectively. The indenter is a perfectly sharp cone. The residual indentation profile from the $10 \mathrm{~nm}$ maximum depth appears to be a magnification of that from the $2.5 \mathrm{~nm}$ maximum depth. The similarity between these residual indentation profiles indicates that the substrate does not have a significant effect on the shape of the residual indentation in this indentation depth range.

Figure 11 shows the hardness as a function of the indentation depth for various $R / h$ values, where $R$ and $h$ denote the tip radius and layer thickness, respectively. The hardness is normalized by the yield stress of the aluminum and the indentation depth is normalized by the layer thickness. In Figs 11(a) and 11(b), the hardness values are based on the plastic depth area and the residual area, respectively. Under the same conditions, these two hardness values are about the same. The fact that the substrate is harder than the layer is reflected in the tendency of the hardness to increase with indentation depths. At large indentation depths, i.e. indentation depths larger than half the layer thickness, the hardness increases with tip radius. This is because a blunt tip induces more substrate plastic deformation and in turn results in larger hardness values. At small indentation depths, the hardness of a homogeneous material decreases with increasing tip radius as shown before. The competition of these two factors make the hardness of $R / h=5$ close to that of $R / h=0$ at an indentation depth of 0.1 of the layer thickness. The same argument can also be used to explain why the hardness is insensitive to the tip radius at indentation depths of about $0.2-0.5$ of the layer thickness. 


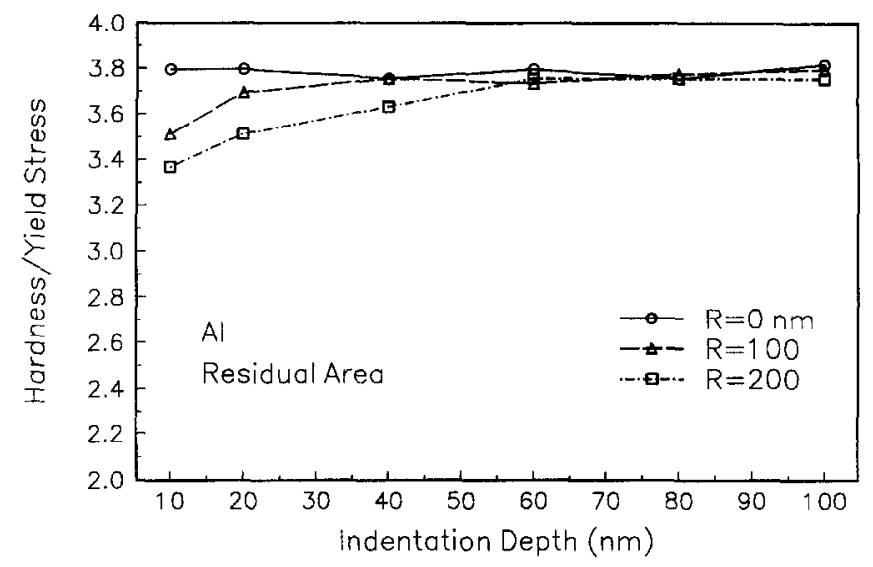

(a)

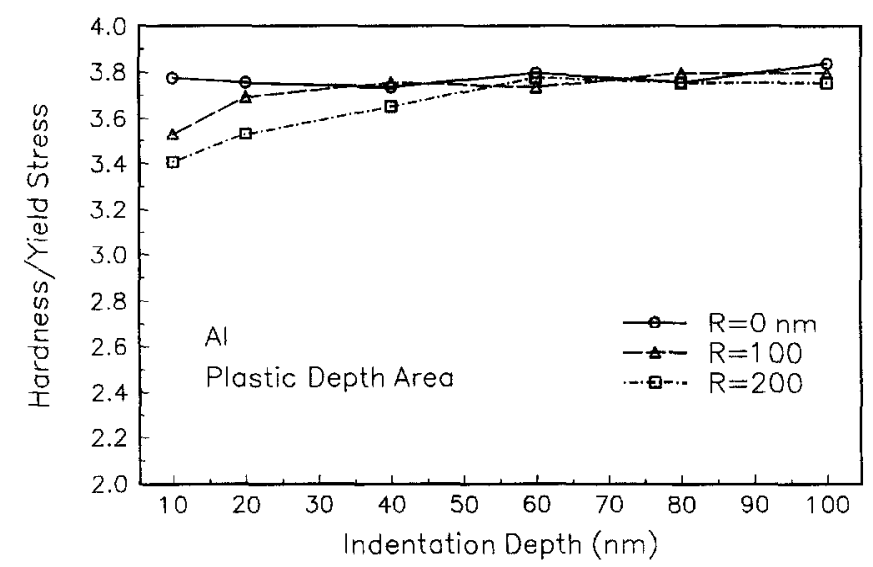

Fig. 8. Hardness versus indentation depth of $\mathrm{Al}$ for various tip radii based on (a) residual area and (b) plastic depth area.

The residual indentation profiles using a perfectly sharp indenter on a $40 \mathrm{~nm}$ thick silicon layer with maximum depths of 4 and $20 \mathrm{~nm}$ are shown in Fig. 12. The shape of the residual indentation profile with maximum depth of $4 \mathrm{~nm}$, one-tenth of layer thickness, is similar to the one on bulk silicon, as can be seen by comparing with Fig. 7(a). There is a relatively small pile-up around the edge of the contact area, which makes it easy to determine the residual area. On the other hand, the residual indentation profile of maximum depth of $20 \mathrm{~nm}$, half of the layer thickness, is very different. This profile consists of two regimes of different slopes connected at point B. The height difference between A and B is about 2 $\mathrm{nm}$, where A denotes the interception of the residual displacement with the $r$-axis. However, the residual area determined from point $A$ is about nine times that from point $B$. The hardness based on the residual area determined from point $\mathrm{A}$ is extremely small. Considering the resolution of the SPM used in our experiments, which is about $0.3 \mathrm{~nm}$, we use the interception between the residual displacement and a horizontal line at $z=0.3 \mathrm{~nm}$ for the determination of the residual area, in order to simulate the hardness tests by the SPM.

The hardness versus indentation depth curves for the silicon layer using plastic depth area and residual area are shown in Figs 13(a) and 13(b) respectively. For the plastic depth area, the hardness decreases smoothly with indentation depth because the substrate is softer than the layer. The hardness at large indentation depths, i.e. indentation depths close to the layer thickness, is not sensitive to the tip radius, which indicates that the substrate effect is dominant in this loading range. The hardness at small indentation depths is sensitive to the tip radius and decreases with increasing tip radius. This is because of two effects; the indenter with larger tip radius may induce more substrate plastic deformation and hence reduce the measured hardness value, and the effect observed on bulk materials. The latter 


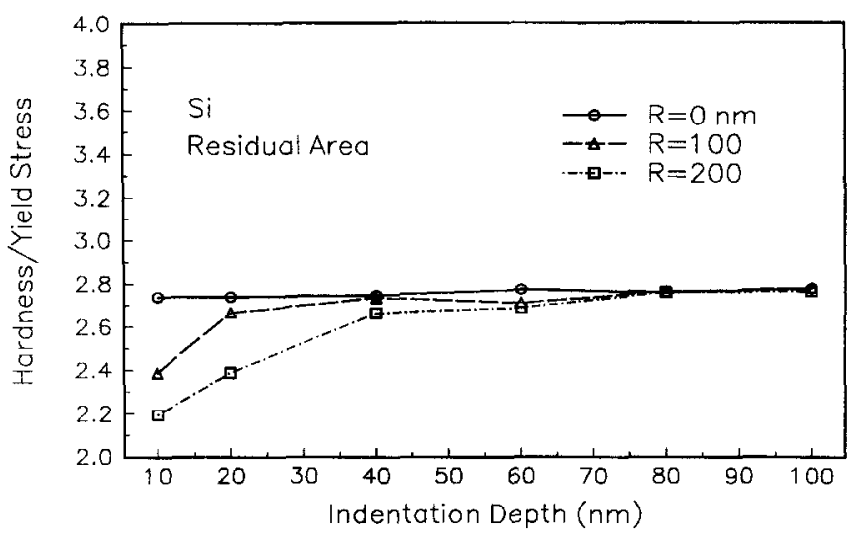

(a)

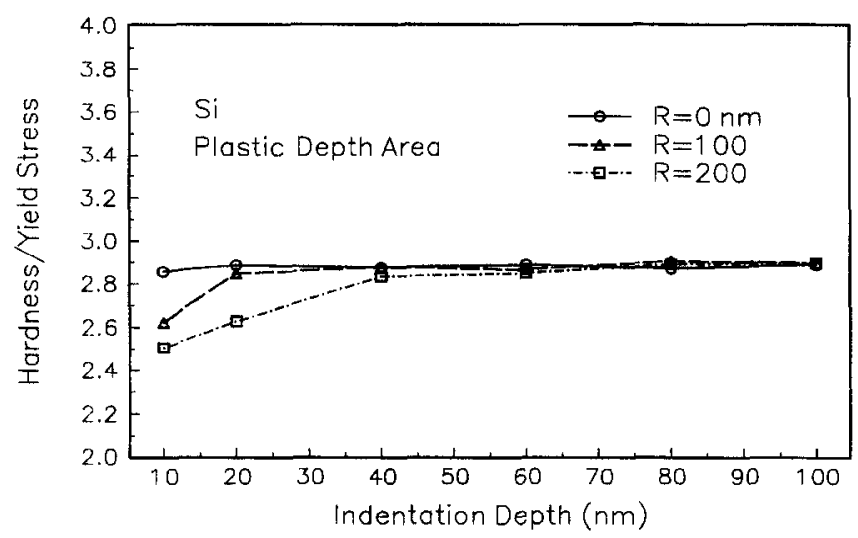

(b)

Fig. 9. Hardness versus indentation depth of Si for various tip radii based on (a) residual area and (b) plastic depth area.

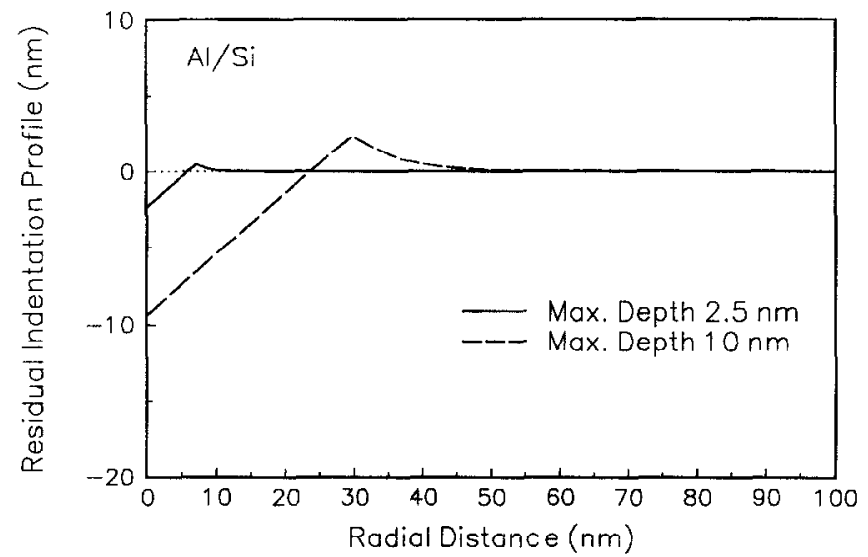

Fig. 10. Residual indentation profile on a $20 \mathrm{~nm}$ thick Al layer at maximum indentation depths of 2.5 and $10 \mathrm{~nm}$. The tip radius is zero.

effect is much smaller than the former. The hardness based on the residual area shows a somewhat different tendency [Fig. 13(b)]. The hardness still decreases with increasing indentation depth. However, the hardness drops suddenly at indentation depths about onethird of the layer thickness and has a small tip-independent value thereafter. This is because of the substrate effect on the residual deformation discussed in the previous paragraph. 


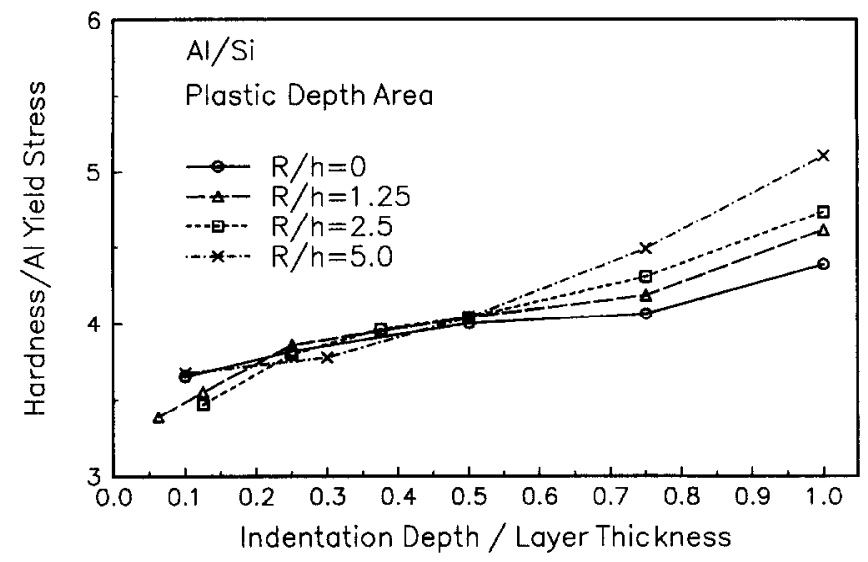

(a)

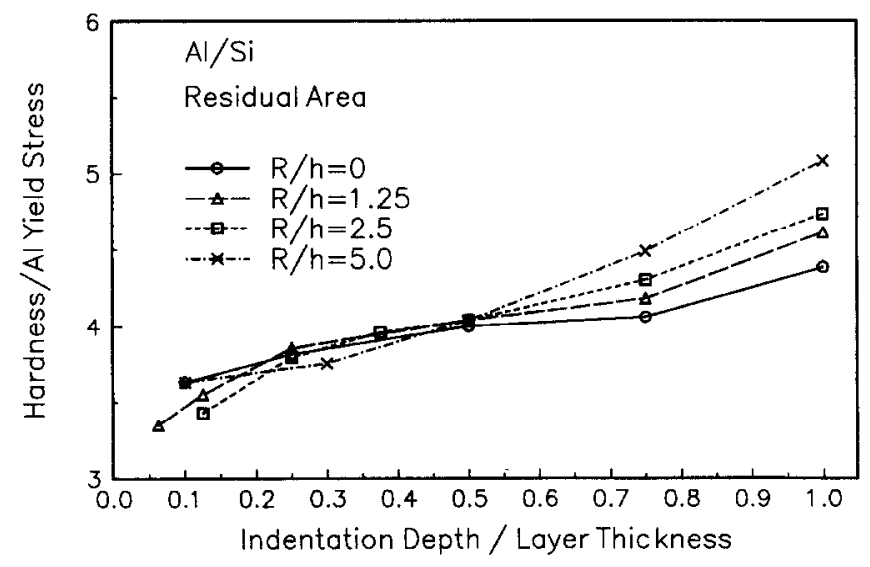

(b)

Fig. 11. Hardness versus indentation depth for an $\mathrm{Al}$ layer on a Si substrate with different tip radii based on (a) plastic depth area and (b) residual area.

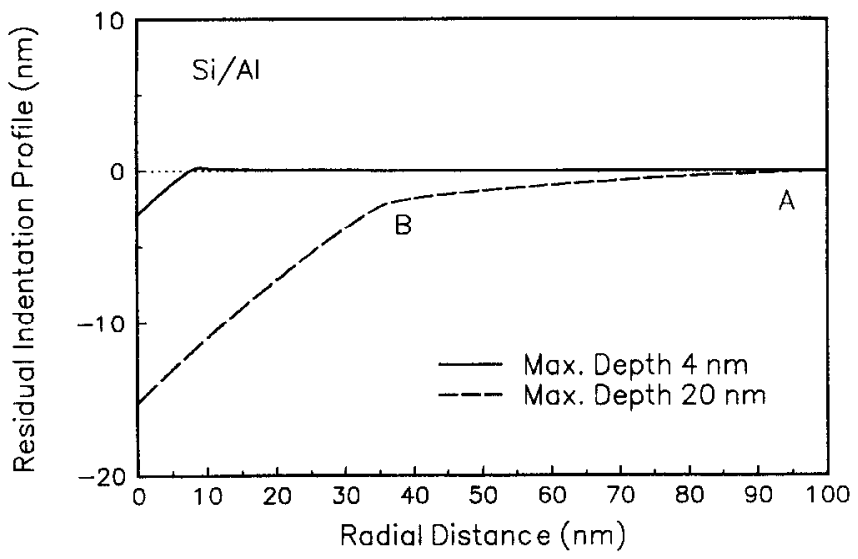

Fig. 12. Residual indentation profile on a $40 \mathrm{~nm}$ thick Si layer at maximum indentation depths of 4 and $20 \mathrm{~nm}$. The tip radius is zero.

For a perfectly sharp indenter, the hardness of the layer obtained at indentation depths of one-fifth the layer thickness is close to that of the bulk material. The difference is less than $10 \%$. For an aluminum layer on a silicon substrate, the hardness obtained at indentation depths of $20 \%$ of the layer thickness can be used as a good approximation of the layer hardness for tip radius to layer thickness ratios of up to 5 . However, for a silicon 


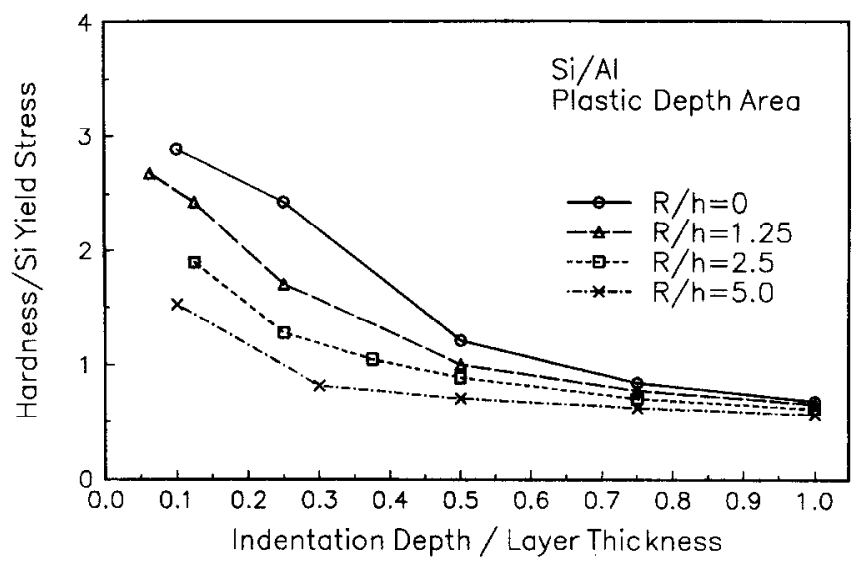

(a)

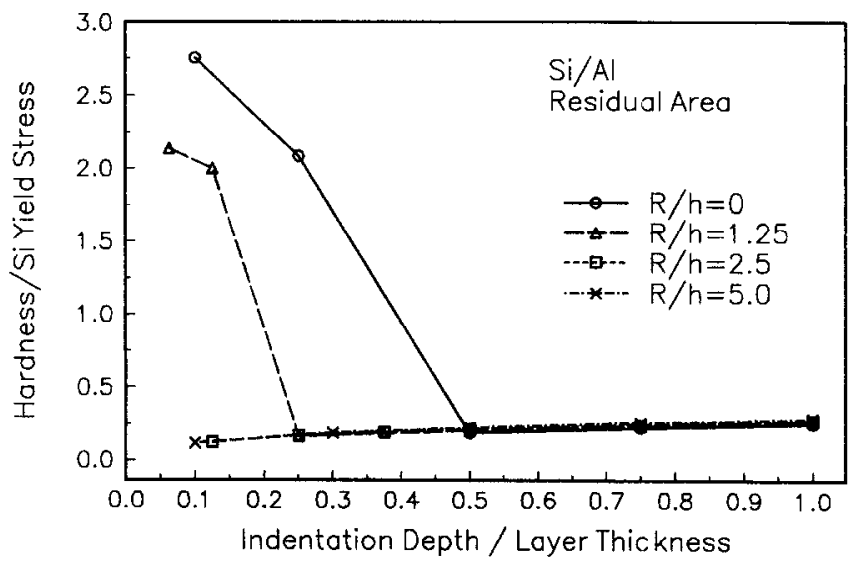

(b)

Fig. 13. Hardness versus indentation depth for a $\mathrm{Si}$ layer on $\mathrm{Al}$ substrate with different tip radii based on (a) plastic depth area and (b) residual area.

layer on an aluminum substrate, hardness is more sensitive to indentation depth and tip radius. At indentation depths of only $10 \%$ of the layer thickness, the hardness is only about one-half of the bulk hardness of the layer material when the tip radius is five times the layer thickness. Hence, it may be very difficult to determine the hardness of a harder layer by using an indenter with a tip radius much larger than the layer thickness. In order to measure the hardness of such a thin layer, the tip radius should be close to or even smaller than the layer thickness.

For a hard layer on a soft substrate ( $\mathrm{Si}$ on $\mathrm{Al}$ ), the hardness bascd on the residual area is more sensitive to the indentation depth than that based on the plastic depth area. From this point of view, the method using a depth-sensing indentation tester may be better than that using SPM technology if the indenter shape can be determined precisely. However, due to the resolution restriction of TEM and replica technology, the smallest depth at which the cross-sectional area of the indenter can be measured is about $20 \mathrm{~nm}$. In other words, for indentation depths less than $20 \mathrm{~nm}$, the corresponding contact area is unknown. Therefore, depth-sensing instruments may not be able to measure the hardness of the current $20-40 \mathrm{~nm}$ thick protective overcoat used in thin film magnetic disks.

\section{CONCLUSION}

Finite element analysis was used to study the tip effect and indentation size effect on nano-indentation hardness tests. The indenter was modeled as a rigid cone with an included angle of $138^{\circ}$ and several tip radii. Two different materials, silicon and aluminum, were 
used to study indentations on bulk material, a hard layer on a soft substrate and a soft layer on a hard substrate. The materials were modeled as elastic-perfectly plastic using von Mises yield criteria. The tip radii and layer thicknesses were chosen to give a variety of radius to thickness ratios of $0,1.25,2.5$ and 5 . Two methods were applied to measure the hardness: one uses the residual area, the other uses the plastic depth area.

Indentation tests on bulk materials show that the residual depth and area are sensitive to the tip radius at small indentation depths. The hardness values obtained using indenters with a nonzero tip radius present a weak tip effect and indentation size effect. For tip radii less than $200 \mathrm{~nm}$ and indentation depths from 0 to $100 \mathrm{~nm}$. the variation of hardness with tip radius and indentation depth is about $10 \%$, which is not significant. In addition, the indentation size effect is opposite to that observed in experiments of nano-indentation tests. This may imply nondislocation mechanisms in nano-indentation tests, as suggested by several researchers.

For a soft layer on a hard substrate, the indentation test is relatively insensitive to either tip radius or indentation depth. Hardnesses obtained at indentation depths of onefifth of the layer thickness are within a 10\% difference in comparison with the bulk hardness of the layer material for tip radius to layer thickness ratios up to 5. On the other hand, for a hard layer on a soft substrate, to obtain a good approximation of the layer hardness at indentation depths of $10 \%$ of the layer thickness, the tip radius to layer thickness ratio should be less than 1.25. The layer is invisible to an indenter with a tip radius much larger than the layer thickness. The critical radius to thickness ratio for measuring the hardness of the layer at an indentation depth of 0.1 of the thickness depends on the properties of the layer and the substrate.

\section{REFERENCES}

ABAQUS Finite Element Program (1989). Jibbitt, Karlsson and Sorensen Inc., Providence RI.

Akyuz, F. A. and Merwin, J. E. (1968). Solution of nonlinear problems of elastoplasticity by finite element method. AIAA J. 6(10), 18251831 .

Belak. J.. Boercker. D. B. and Stowers, I. F. (1993). Simulation of nanometer-scale deformation of metallic and ceramic surfaces. MRS Bulletin 18, 55-60.

Bhattacharya, A. K. and Nix, W. D. (1988a). Finite element simulation of indentation experiments. Int. J. Solids Siructures 24(9), 881-891.

Bhattacharya, A. K. and Nix, W. D. (1988b). Analysis of elastic and plastic deformation associated with indentation testing of thin films on substrates. Int. J. Solids Structures 24(12), 1287-1298.

Bhattacharya. A. K. and Nix, W. D. (1991). Finite element analysis of cone indentation. Int. J. Solids Structures 27(8), 1047-1058.

Doerner M. F. and Nix. W. D. (1986). A method for interpreting the data from depth-sensing indentation instruments. J. Mater. Res. 1(4), 601-609.

Follansbee, P. S. and Sinclair. G. B. (1984). Quasi-static normal indentation of an elasto-plastic half-space by a rigid sphere -I. Analysis. Int. J. Solids Structures 20(1), $81-91$.

Gane, N. and Bowden. F. P. (1968). Microdeformation of solids. J. Appl. Phys. 19(2), 1432-1435.

Hardy, C. Baronet, C. N. and Tordion, G. V. (1971). The elasto-plastic indentation of a half-space by a rigid sphere. Int. J. Numer. Meth. Engng 3, 451-462.

Johnson, K. L. (1985). Contact Mechanics. Cambridge University Press, Cambridge, UK.

Komvopoulos. K. (1989). Elastic--plastic finite element analysis of indented layered media. ASME J. Trib. 111(3), 430-439.

Kral, E. R., Komvopoulos, K. and Bogy, D. (1993). Elastic-plastic finite element analysis of repeated indentation of a half-space by a rigid sphere. ASME J. Appl. Mech. 60, 829-841.

Lu. C.-J., Bogy, D. B. and Kancko, R. (1994). Nanoindentation hardness tests using a point contact microscope. ASME J. Tribol. 116, 175-180.

Oliver, W. C. and Pharr, G. M. (1992). An improved technique for determining hardness and elastic modulus using load and displacement sensing indentation experiments. J. Mater. Res. 7(6), 1564-1583.

Pethica, J. B., Hutchings, R. and Oliver, W. C. (1983). Hardness measurement at penetration depth as small as $20 \mathrm{~nm}$. Phil. Mag. A48, 593-606.

Pharr. G. M. and Oliver W. C. (1989). Nanoindentation of silver-relations between hardness and dislocation structure. J. Mater. Res. 4(1), 94-101.

Shih. C. W., Y and M. and Li, J. C. M. (1991). Effect of tip radius on nanoindentation. J. Mater. Res. 6(12), 2623.2628 .

Tabor. D. (1951). The Hardness of Metals. Oxford University Press, Oxford.

Wierenga, P. E. and Franken. A. J. J. (1984). Ultra-microindentation apparatus for the mechanical characterization of thin films. J. Appl. Phys. 55(12), $4244-4247$

Yanagisawa, M. and Motomura. Y. (1987). An ultra-microindentation hardness tester and its application to thin films. Lubr. Engng 43(1), 52-56. 